\title{
Les prescriptions anticipées d'imagerie par les infirmier(e)s diplômé(e)s d'État en structure des urgences
}

\author{
The Nurse-Initiated X-Ray Orders in Emergency Department
}

\author{
P.-G. Claret $\cdot$ X. Bobbia $\cdot$ J.-E. de La Coussaye \\ (C) SFMU et Lavoisier SAS 2017
}

Le triage est nécessaire lorsque les soignants ne peuvent répondre immédiatement aux besoins des patients et fait partie intégrante de l'organisation d'un service d'urgence (SU). Il est défini comme la hiérarchisation des prises en charge des patients en fonction de leur gravité. Cette définition implique des conséquences individuelles et collectives au triage. En effet, les objectifs du triage sont multiples. Ils sont de prendre en charge au mieux chaque patient, mais aussi d'organiser efficacement le système de soins. L'intérêt du triage et le rôle de l'infirmier(e) d'accueil et d'orientation (IAO) deviennent ainsi grandissants à mesure que la surcharge de nos SU augmente et avec elle le temps d'attente. À partir de ce constat, l'équipe de l'hôpital Tenon a souhaité d'une part renforcer le rôle de l'IAO et d'autre part utiliser ce temps d'attente précédant le premier contact médical. Dans ce numéro des Annales Françaises de Médecine d'Urgence, cette équipe nous présente donc une étude monocentrique de type avant-après dont l'objectif est de déterminer l'impact de la demande anticipée de radiographies par l'IAO sur le temps de passage des patients en SU [1]. Les auteurs observent que le temps d'attente avant premier contact médical a diminué de 41 minutes entre la phase avant et la phase après $(\mathrm{p}=0,008)$, de même que la durée de séjour en SU (48 minutes ; $p<0,001)$. Ainsi, le temps d'attente n'est plus un temps mort source d'insatisfaction pour le patient, mais il devient une étape de la démarche diagnostique. L'utilité du protocole anticipé est ainsi démontrée en termes de temps de passage.

Encore faut-il discuter de la faisabilité de ces protocoles anticipés. Sur le sujet, la littérature nous procure des éléments de réponse : après une formation bien conduite (et

P.-G. Claret $(\bowtie) \cdot$ X. Bobbia $\cdot$ J.-E. de La Coussaye

Pôle anesthésie réanimation douleur urgences, centre hospitalier universitaire de Nîmes, 4 rue du Professeur Robert Debré, F-30029 Nîmes, France

e-mail : pierre.geraud.claret@gmail.com

J.-E. de La Coussaye

Université Montpellier-Nîmes, 2 rue de l'École de Médecine,

F-34060 Montpellier, France surtout réitérée) à la traumatologie légère, les infirmier(e)s diplômé(e)s d'État (IDE) demandent pertinemment les radiologies nécessaires au diagnostic. Ainsi, Patel et al. [2] ont étudié la pertinence et les effets d'une prescription anticipée d'imagerie chez 206 patients se présentant en SU pour un traumatisme du membre inférieur. Ces 206 patients ont fait des radiologies standard prescrites par des IAO (101 patients) ou des médecins urgentistes (105 patients). Si dans cette étude, la durée moyenne de séjour en SU n'était pas différente entre les groupes IAO et médecins (159 vs 173 minutes), probablement du fait d'un manque de puissance de l'étude, le taux de fractures retrouvées était comparable entre les groupes IAO et médecins ( 35 vs $27 \%$ ). L'importance d'une formation répétée a été soulignée par Valentian et al. [1] dans la discussion de leur article. De même, une récente étude [3] publiée en 2016 en Australie montre que l'évaluation de ces pratiques, et surtout la reformation des équipes paramédicales, améliore le respect des protocoles anticipés de prescription d'imagerie par les IDE (48 vs $90 \% ; \mathrm{p}<0,001)$. Ces améliorations portaient principalement sur la qualité de l'interrogatoire (mécanisme du traumatisme et localisation).

En plus de leur utilité et de leur faisabilité, les protocoles anticipés permettent une valorisation du travail des IDE, de leurs compétences, mais aussi de leur expérience. En effet, l'équipe de Stiell et al., à l'origine de la règle d'Ottawa, a publié une étude dont l'objectif était de déterminer les éléments facilitateurs ou bloquants pour appliquer un protocole anticipé aidant à retirer par les IDE les colliers cervicaux aux patients victimes d'un traumatisme [4]. Cette enquête montre que les IDE sont grandement satisfaits $(96 \%)$ de ces protocoles. La plus importante barrière pour appliquer ces protocoles était la surcharge de travail induite (37\%), ce qui ne semble pas être le cas dans l'étude de Valentian et al. [1]. Bien sûr, d'autres études multicentriques ou avec un effectif plus important devront confirmer la validité du travail de l'équipe de l'hôpital Tenon. Mais l'étude de Valentian et al. [1] a le grand mérite de montrer ce que certaines SU font souvent sans évaluation ni cadre réglementaire. En 
effet, certaines activités attribuées aux IDE le sont parfois hors cadre juridique ou hors protocole (confection de plâtre, prescription anticipée d'antalgiques ou d'imagerie). Il existe pourtant un cadre aux protocoles de coopération qui doivent être validés par l'Agence Régionale de Santé et ce transfert de tâche sous-entend de pouvoir recourir en permanence à un médecin référent au sein du service. Les protocoles anticipés sont donc utiles et valorisants, mais une réflexion s'impose avant de les mettre en place, notamment sur leur application en fonction de la réglementation française.

Liens d'intérêts : Les auteurs déclarent ne pas avoir de lien d'intérêt.

\section{Références}

1. Valentian M, Mewasing IB, Burggraff E, et al (2017) Intérêt d'une demande anticipée de radiographies par l'infirmière organisatrice de l'accueil, dans les traumatismes de cheville. Ann Fr Med Urg 7:77-84

2. Patel H, Celenza A, Watters T (2012) Effect of nurse initiated Xrays of the lower limb on patient transit time through the emergency department. Australas Emerg Nurs J 15:229-34

3. Thompson N, Murphy M, Robinson J, Buckley T (2016) Improving nurse initiated $\mathrm{X}$-ray practice through action research. J Med Radiat Sci 63:203-8

4. Clement CM, Stiell IG, Davies B, et al (2011) Perceived facilitators and barriers to clinical clearance of the cervical spine by emergency department nurses: A major step towards changing practice in the emergency department. Int Emerg Nurs 19:44-52 